\title{
Properties of Dynamic Earthquake Ruptures With Heterogeneous Stress Drop
}

\author{
J.-P. Ampuero, J. Ripperger, P. M. Mai \\ Institute of Geophysics, ETH Zürich, Switzerland
}

Earthquake rupture is a notoriously complex process, at all observable scales. We introduce a simplified semi-dynamic crack model to investigate the connection between the statistical properties of stress and those of macroscopic source parameters such as rupture size, seismic moment, apparent stress drop and radiated energy. Rupture initiation is treated consistently with nucleation on a linear slipweakening fault, whereas rupture propagation and arrest are treated according to the Griffith criterion. The available stress drop is prescribed as a spatially correlated random field and is shown to potentially sustain a broad range of magnitudes. By decreasing the amplitude of the stress heterogeneities or increasing their correlation length the distribution of earthquake sizes presents a transition from GutenbergRichter to characteristic earthquake behavior. This transition is studied through a mean-field analysis. The bifurcation to characteristic earthquake behavior is sharp, reminiscent of a first-order phase transition. A lower roll-off magnitude observed in the Gutenberg-Richter regime is shown to depend on the correlation length of the available stress drop, rather than being a direct signature of the nucleation process. More generally, we highlight the possible role of the stress correlation length scale on deviations from earthquake source self-similarity. The present reduced model is a building block towards understanding the effect of structural and dynamic fault heterogeneities on the scaling of source parameters and on basic properties of seismicity.

\section{INTRODUCTION}

Kinematic source inversions indicate that earthquake rupture is complex on a broad range of length-scales [Mai and Beroza, 2002]. However the details of fault dynamic weakening are beyond their intrinsic resolution limits [Guatteri and Spudich, 2000], which is in contrast with the high resolution of modern dynamic earthquake simulations. This warrants the introduction of more elementary rupture models that can nevertheless elucidate essential aspects of earthquake complexity. In linear elastic fracture dynamics

Earthquakes: Radiated Energy and the Physics of Faulting Geophysical Monograph Series 170

Copyright 2006 by the American Geophysical Union. 10.1029/170GM25 (subshear) rupture is controlled by two parameters: stress drop and fracture energy [Freund, 1998; Husseini et al, 1975]. Both can be non uniform. Slip distributions inferred from seismological and geodetic data provide a valuable constraint on possible stochastic parameterizations of stress drop [Mai and Beroza, 2002; Lavallée and Archuleta, 2003]. Fracture energy might be the only dynamic parameter that can be robustly inferred from frequency-limited strong motion data [Guatteri and Spudich, 2000].

Rupture under non uniform stress or strength has been previously studied through numerical simulation [Day, 1982; Boatwright and Quin, 1986; Oglesby and Day, 2002] or statistical approaches [Heimpel, 1996; Rundle et al, 1998]. It is still important for strong ground motion prediction and for studies of source scaling to understand how the 
heterogeneity of mechanical fault parameters and initial conditions leads to variability of dynamic rupture properties. A computationally intensive approach consists on the statistical analysis of a large number of dynamic simulations with stochastic fault properties [Ripperger et al, 2005]. As a preliminary step we explore here a simplified model based on fracture mechanics with emphasis on the effects of heterogeneous stress drop. Our aim is to identify and quantify the aspects of the spatial distribution of stres drop that control the overall tendency and variability of macroscopic source properties such as earthquake size, average rupture velocity, apparent stress drop, seismic moment and radiated energy.

Here stress drop heterogeneities are mapped onto initial stress heterogeneities that mimic the result of prior seismicity and are prescribed as correlated stochastic fields parameterized by a few statistical quantities. We explore the parameter space using an efficient semi-dynamic model, formulated in the next section, that despite its simplicity encapsulates the main ingredients. In Section 3 a transition from GutenbergRichter (GR) to characteristic-earthquake (CE) frequencysize statistics is identified. The role of the stress correlation length on the scaling of source properties is highlighted and explained through a mean-field analysis of crack arrest. These results and further extensions are discussed in Section 4.

\section{MODEL ASSUMPTIONS}

\subsection{Stress Drop}

We consider a 1D antiplane fault with coordinate $x$ along strike. Linear slip-weakening friction is assumed, with uniform yield strength $\tau_{p}$, dynamic strength $\tau_{d}$ and characteristic slip-weakening distance $D_{c}$. Neglecting dynamic overshoot and undershoot, the final stress is fixed to $\tau_{d}$. The stress $\tau_{0}(x)$ at the onset of an earthquake results from the large scale tectonic stressing and from the previous seismicity contributing over a broad range of scales. Dynamic crack propagation is primarily sensitive to stress drop $\Delta \tau(x)=\tau_{0}(x)-\tau_{d}$, strength excess $\tau_{d}-\tau_{0}(x)$ being essential only during nucleation and inplane super-shear transition. Simulating 3D seismic cycles in well resolved continuum models is still at the edge of our computational capabilities. Alternatively we can draw hypothetical stress fields from a statistical distribution, characterized by a few parameters, and study the properties of the resulting earthquake ruptures. [Mai and Beroza, 2002] found that coseismic slip distributions from finite-fault source inversions can be described as random fields with von Karman auto-correlation function. Our focus here is on correlated spatial distributions of available stress drop, with well defined standard deviation $s t d$ and correlation length $a_{c}$. We consider normally distributed fields with truncated power law spectrum:

$$
\Delta \tau(k) \propto s t d\left(a_{c}^{-2}+k^{2}\right)^{-(H / 2+1 / 4)}
$$

where $k$ is the along strike wavenumber and $H$ the roughness (Hurst) exponent. If $H>0$, the auto-correlation function is well defined:

$$
C(x)=\frac{\left(x / a_{c}\right)^{H} K_{H}\left(x / a_{c}\right)}{2^{H-1} \Gamma(H)}
$$

where $K_{H}$ is the modified Bessel function of the second kind, of order $H$, and $\Gamma$ is the Gamma function. The mean value of $\Delta \tau(x)$ is not prescribed arbitrarily but results from the nucleation process, as described in the next section.

\subsection{Nucleation Criterion}

In previous numerical studies [e.g. Oglesby and Day, 2002] the hypocenter location has been treated as a free parameter. Here, instead, it is determined in consistency with a mechanical analysis of nucleation. Instabilities on linear slip-weakening faults are preceded by aseismic slip, driven by tectonic load, in regions where stress overcomes the yield strength. As shown by [Uenishi and Rice, 2003], this stable nucleation stage ends when the half-size of the slipping zone reaches the critical length

$$
a_{v}=0.5789 \frac{\mu D_{c}}{\tau_{p}-\tau_{d}}
$$

This length is "universal" in the sense that it is independent of the spatial distribution of the initial stress $\tau_{0}(x)$. This result assumes that aseismic slip remains smaller than $D_{c}$ and that stress peaks are well separated leading to weakly interacting nucleation sites. Both requirements are satisfied in particular when $a_{c} \gg a_{v}$.

The uniform stress increase $\tau_{c}$ required to reach instability and the location of the center of the nucleation zone are given by the minimum of a filtered version of the strength excess:

$$
\tau_{c}=\min _{x}\left[\left(\tau_{p}-\tau_{0}\right) * \Phi_{c}\right]
$$

where $*$ denotes space convolution. The "nucleation filter" $\Phi_{c}$ is the first eigenfunction of the elastostatic problem, rescaled to $a_{v}$ and normalized to unit average. For all practical purposes it is approximated by

$$
\Phi_{c}(\xi)=\left(0.6944-0.2312 \xi^{2}\right) \sqrt{1-\xi^{2}}
$$

for $|\xi| \leq 1$, where $\xi=x / a_{v}$ [Uenishi and Rice, 2003]. The nucleation location and $\tau_{c}$ are insensitive to stress heterogeneities of length scales much shorter than $a_{v}$. 
The stress drop $\Delta \tau$ available for the ensuing earthquake is inherited from the nucleation process. For $a_{c} \gg a_{v}$, the aseismic stress drop can be neglected and

$$
\begin{aligned}
& \Delta \tau(x) \approx \tau_{0}(x)+\tau_{c}-\tau_{d}= \\
& \tau_{p}-\tau_{d}+\tau_{0}(x)-\max \left[\tau_{0} * \Phi_{c}\right]
\end{aligned}
$$

Note that the contribution from the last two terms is proportional to $s t d$. We further restrict our attention to bilateral ruptures by symmetrizing each stress drop field with respect to its nucleation point, relocated at $x=0$ without loss of generality.

\subsection{Propagation and Arrest Criteria}

Dynamic rupture begins with an initial slip acceleration stage [Campillo and Ionescu, 1997; Ampuero et al, $2002]$ that we will not consider in our model. Soon after, crack-like rupture develops and the size of the process zone becomes small enough to warrant a small scale yielding approximation. The details of the friction law can then be ignored and rupture is governed by the balance between the energy release rate $G$ flowing towards the crack tip and fracture energy, $G_{c}=\left(\tau_{p}-\tau_{d}\right) D_{c} / 2$ for linear slip-weakening. For a mode III crack of half-size $a$, rupture velocity $V_{R}$ and stress drop $\Delta \tau(x)$ :

$$
G(v, a, \Delta \tau)=\sqrt{\frac{1-V_{R} / c_{S}}{1+V_{R} / c_{S}}} G^{*}(a, \Delta \tau)
$$

with

$$
G^{*}(a, \Delta \tau)=\frac{K^{* 2}}{2 \mu}
$$

where $c_{s}$ is shear wave velocity, $\mu$ shear modulus and $K^{*}$ the stress intensity factor that would prevail immediately after rupture arrest. Following the Griffith criterion, the rupture propagates with $G=G_{c}$, which provides a "crack tip equation of motion" [Freund, 1998], and stops if $G^{*} \leq G_{\text {c }}$.

In general $K^{*}$ is a convoluted function of crack growth history $a(t)$ and stress drop $\Delta \tau(x)$, especially complicated by the interaction between the two crack tips [Rose, 1976; Leise and Walton, 2001]. After rupture arrest waves multiply diffracted at the crack tips bring $K^{*}$ progressively, with oscillations, to its static value $K_{0}$. For symmetric non uniform stress drop:

$$
K_{0}(a)=\sqrt{\pi a} \frac{2}{\pi} \int_{0}^{a} \frac{\Delta \tau(x)}{\sqrt{a^{2}-x^{2}}} d x
$$

A "semi-dynamic" approximation combines equations (7) and (8) with the assumption $K^{*} \approx K_{0}$. Although exact only for semi-infinite straight cracks, it has been applied with success to dynamic fracture problems involving branching and crack-microcrack interactions [Bouchbinder et al,
$2004 ; 2005]$. For our purposes, this approximation encapsulates the main dependency on stress drop heterogeneity, as will be illustrated later by favorable comparisons to fully dynamic 3D simulations. The criterion for crack arrest becomes

$$
G_{0} \doteq \frac{K_{0}^{2}}{2 \mu}=G_{c} \text { and } \frac{d G_{0}}{d a}<0
$$

\section{RUPTURE PROPERTIES IN STOCHASTIC STRESS DROP FIELDS}

For each realization of $\Delta \tau(x), K_{0}(a)$ is computed by numerical integration of equation (9). The final earthquake size is determined as the crack size $a$ at which $G_{0}(a)$ becomes smaller than $G_{c}$. Figure 1 shows the distribution of earthquake sizes obtained at fixed $H$ and $a_{c}$ for different values of std (normalized by strength drop $\tau_{p}-\tau_{d}$ ). Each curve contains $10^{4}$ realizations of the heterogeneous stress field. The seismic moment is computed as [Madariaga, 1979]

$$
M_{0}=2 \int_{0}^{a} \Delta \tau(x) \sqrt{a^{2}-x^{2}} d x
$$

Note that in this 2D model $M_{0}$ is a seismic moment per unit of out-of-plane length and has units of force instead of newtonmeters. For high values of $s t d$ the frequency-size distribution is reminiscent of a Gutenberg-Richter distribution (GR), with fast decay at large magnitudes and a lower roll-off moment $M^{*}$. At lower std we observe instead a characteristic-earthquake distribution (CE), with event sizes dominated by the highest magnitude, ultimately determined by the finite size of the modelled fault segment. To understand this transition we analyze the mean-field properties of the model.

Figure 2-a shows the stress drop distribution stacked over $10^{4}$ realizations as a function of hypocentral distance. Assuming $a_{c} \gg a_{v}$ this ensemble-averaged stress drop is related to the the auto-correlation function (2) by

$$
\Delta \tau(x) \approx \tau_{p}-\tau_{d}+m \operatorname{std}\left[C\left(x / a_{c}\right)-1\right]
$$

where $m$ is a slowly decreasing function of the ratio between $a_{c}$ and whole fault size. The associated ensemble-averaged stress intensity factor (Figure 2-b) is

$$
\bar{K}_{0}(a) \approx \sqrt{\pi a}\left[\tau_{p}-\tau_{d}+m s t d\left(\frac{2}{\pi} \int_{0}^{a} \frac{C\left(x / a_{c}\right)}{\sqrt{a^{2}-x^{2}}} d x-1\right)\right]
$$

For cracks growing far beyond the correlation length, following [Dyskin, 1999] the behavior of $\bar{K}_{0}(a)$ is best understood as the competition between a uniform background stress 

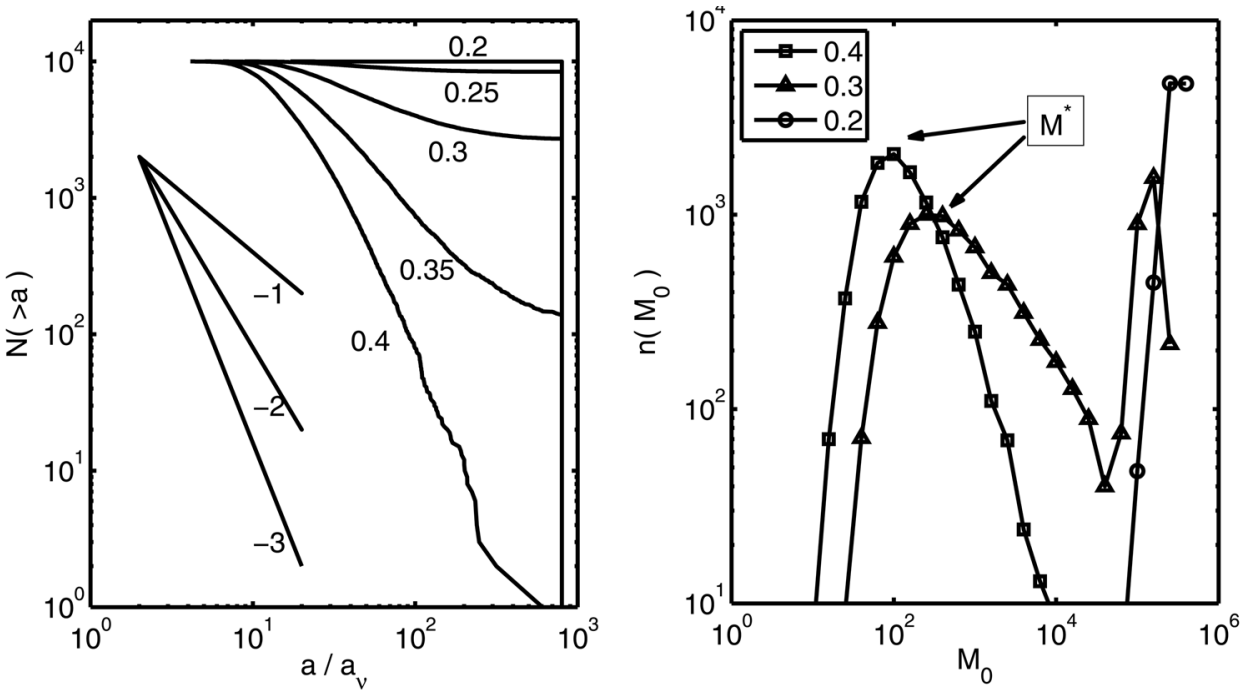

Figure 1. Frequency-size statistics in the semi-dynamic model: cumulative crack size distributions (left) and seismic moment histograms (right). Each curve contains $10^{4}$ realizations of the stochastic stress field, with $H=1, a_{c}=5 a_{v}$ and different values of $s t d$ as indicated by labels (normalized by $\tau_{p}-\tau_{d}$ ). The straight lines on the left are visual guides for power law distributions with exponents as labeled. A transition from GR-like to CE-like behavior is observed. The lower roll-off moment $M^{*}$ of the GR is higher for smaller std and is related to both the nucleation length $a_{v}$ and the correlation length $a_{c}$.

drop, $\Delta \bar{\tau}=\tau_{p}-\tau_{d}-m$ std, and a pair of tangential point forces, $F=m$ std $a_{c} \int_{0}^{\infty} C(\xi) d \xi$, located at the crack center:

$$
\bar{K}_{0}(a) \approx \sqrt{\pi a}\left(\Delta \bar{\tau}+\frac{2}{\pi} \frac{F}{a}\right)
$$

Note how these two contributions have opposite dependencies on both $a$ and $s t d$. For large enough std the arrest criterion (10) applied to $\bar{K}_{0}$ leads to a typical crack size $a^{*}$ that corresponds to the roll-off moment of Figure 1. If std is reduced the background stress drop $\Delta \tau$ becomes higher and $F$ smaller, $\bar{K}_{0}(a)$ curves upwards and $a^{*}$ increases (Figure 2-b). Above a critical value $s d_{c}$, for which

$$
F \Delta \bar{\tau}=\mu G_{c} / 4
$$

the arrest condition can no longer be met and ruptures run away, breaking the whole fault length. This transition is better visualized in Figure 2-c in terms of the total energy change

$$
\Delta E=\int_{a_{v}}^{a}\left[G_{c}-G_{0}\left(a^{\prime}\right)\right] d a^{\prime}
$$

The Griffith criterion for a stable equilibrium crack (10) is equivalent to a minimization of $\Delta E$ with respect to $a$. For large std there is a distinct energy minimum corresponding to the stable equilibrium at crack arrest (point A in Figure 2). At a larger $a$ there is an energy maximum corresponding to an unstable equilibrium state (point B). As std decreases the two equilibria collapse (point $\mathrm{C}$ ) and disappear. The typical event size $a^{*}$ does not diverge continuously at the transition but jumps to $\infty$ from a finite critical size (Figure 2-d). From the condition (15) the critical size is found to scale as

$$
a^{*} \propto \frac{a_{c}^{2}}{a_{v}}
$$

and the critical std as

$$
s t d_{c} \approx\left(\tau_{p}-\tau_{d}\right) / m
$$

Two important source quantities are plotted in Figure 3, for a fault in the GR regime. An apparent stress drop is defined with reference to a constant stress drop crack:

$$
\Delta \tau^{\prime}=M_{0} /\left(\pi / 2 a^{2}\right)
$$

(Note again that $M_{0}$ is in newtons.) Stress drop shows a tendency to decrease with moment, especially for earthquake sizes comparable to the correlation length $a_{c}$. This is expected from nucleation in regions of high stress. If stress is assumed constant $\left(=\tau_{d}\right)$ after the passage of the rupture front radiated energy is related to the total energy change during rupture (16) by [Freund, 1998; Husseini and Randall, 1976]:

$$
E_{R}=-\Delta E
$$


a)

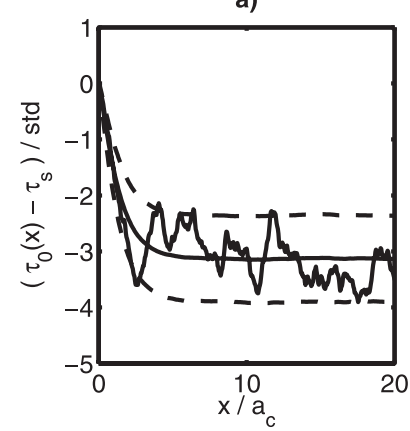

c)

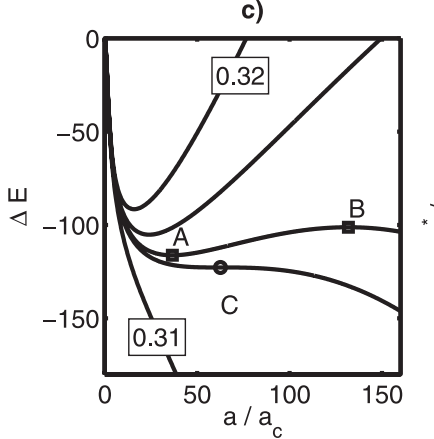

b)

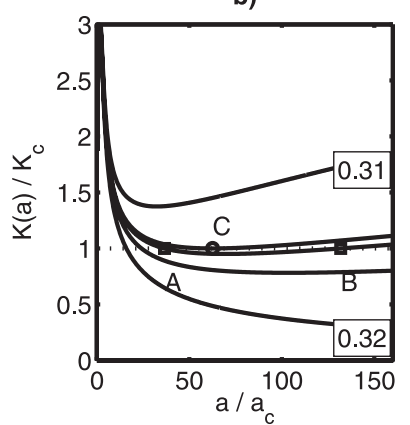

d)

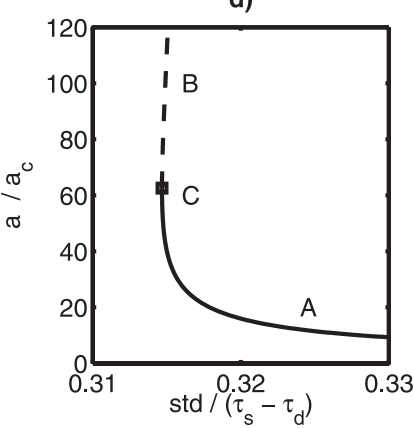

Figure 2. (a) Initial stress distribution as a function of hypocentral distance: typical realization (rough solid line), ensemble-average (smooth solid curve) with standard deviation (dashed curves). (b) Ensemble-averaged stress intensity factor and (c) total energy change as a function of crack size for some values of std within the range indicated by labels. Points $\mathrm{A}, \mathrm{B}$ and $\mathrm{C}$ are stable, unstable and critical equilibria respectively, they verify $K=K_{c}$ and are energy extrema. As std is reduced, A and B collapse into C, then disappear. (d) Crack length $a^{*}$ corresponding to mean-field stable (solid curve, A) and unstable (dashed curve, B) equilibria as a function of $s t d$. The stable crack size is associated to the roll-off moment $M^{*}$ of the frequency-size statistics. A discontinuous transition occurs at a critical std (point C).

Radiated energy is overall constant in the logarithmic scale of Figure 3-b, although some weak tendencies are observed at the lowest $\left(a<a_{v}\right)$ and largest magnitudes $\left(a>a_{c}\right)$. The scale-dependency of these quantities is a combined signature of the correlation length $a_{c}$ and the nucleation length $a_{v}$.

\section{DISCUSSION}

The statistical properties of $\Delta \tau(x)$ may be non stationary during the earthquake cycle. In models of seismicity featuring intermittent criticality the variability and correlation length increases as a large event approaches. As we make no attempt to describe the evolution of seismicity our analysis relates to ensemble statistics over snapshots of fault zones taken at a fixed stage of their cycle. In this way we identify intrinsic statistical features for given std and $a_{c}$, which can be useful to interpret more complete models of seismicity. These intrinsic statistics may be directly observable over a limited time window only if the time-scale of non-stationarity is longer.

Fracture energy has been often understood as a material property although the paradigm of scale-dependent $G_{c}$ has resurrected recently. A recurrent claim is that a constant $G_{c}$ leads inevitably to run-away cracks, which is incompatible with the GR distribution of earthquakes sizes. This argument holds for uniform stress drop but may not be reasonable for events breaking on natural faults that have sustained prior seismicity. The present model illustrates how a broad distribution of magnitudes can be generated solely by stress heterogeneities, without requiring a systematic scale-dependent $G_{c}$. In turn, assuming for instance $G_{c} \propto a$ favors rupture arrest and promotes larger apparent stress drops. Statistical properties of rupture arising from uncorrelated heterogeneities of $G_{c}$ have been studied by [Heimpel, 1996] and remain to be merged with the results of the present study.

A roll-off in frequency-size distributions at a low moment $M^{*}$ is usually associated to catalog incompleteness. However, in dense borehole and deep mine monitoring networks $M^{*}$ has been suggested to be above the detection threshold [Heimpel and Malin, 1998; Richardson and Jordan, 2002] and has been interpreted as a signature of a minimal nucleation size $a_{v}$, yielding an estimate of $D_{c}$ in the high end of the laboratory range or much higher. Our analysis indicates that $M^{*}$ may strongly depend on the correlation length of stress. The associated source size $a^{*}$ can be much larger than $a_{v}$ and, in the absence of an independent estimate of $a_{c}$, it cannot be mapped directly into a nucleation length.

The transition from GR to CE frequency-size statistics in the present semi-dynamic 2D model is relevant to understand some features of fully dynamic 3D simulations. Figure 4-left shows three ruptures simulated with the same stress drop distribution but different standard deviation std. At a critical
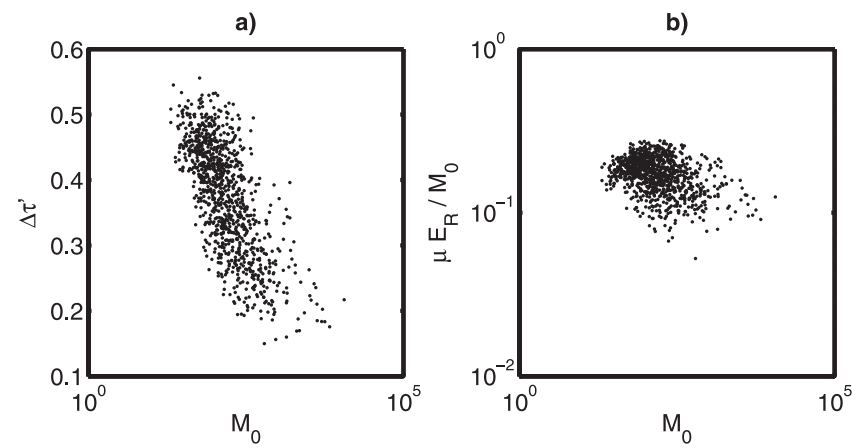

Figure 3. Apparent stress drop (a) and apparent stress (b) for $10^{3}$ events with $s t d=0.4$. All stresses are scaled by $\tau_{p}-\tau_{d}$. The observed magnitude-dependence, stronger for earthquake sizes comparable to the stress correlation length, is due to nucleation near stress peaks. 

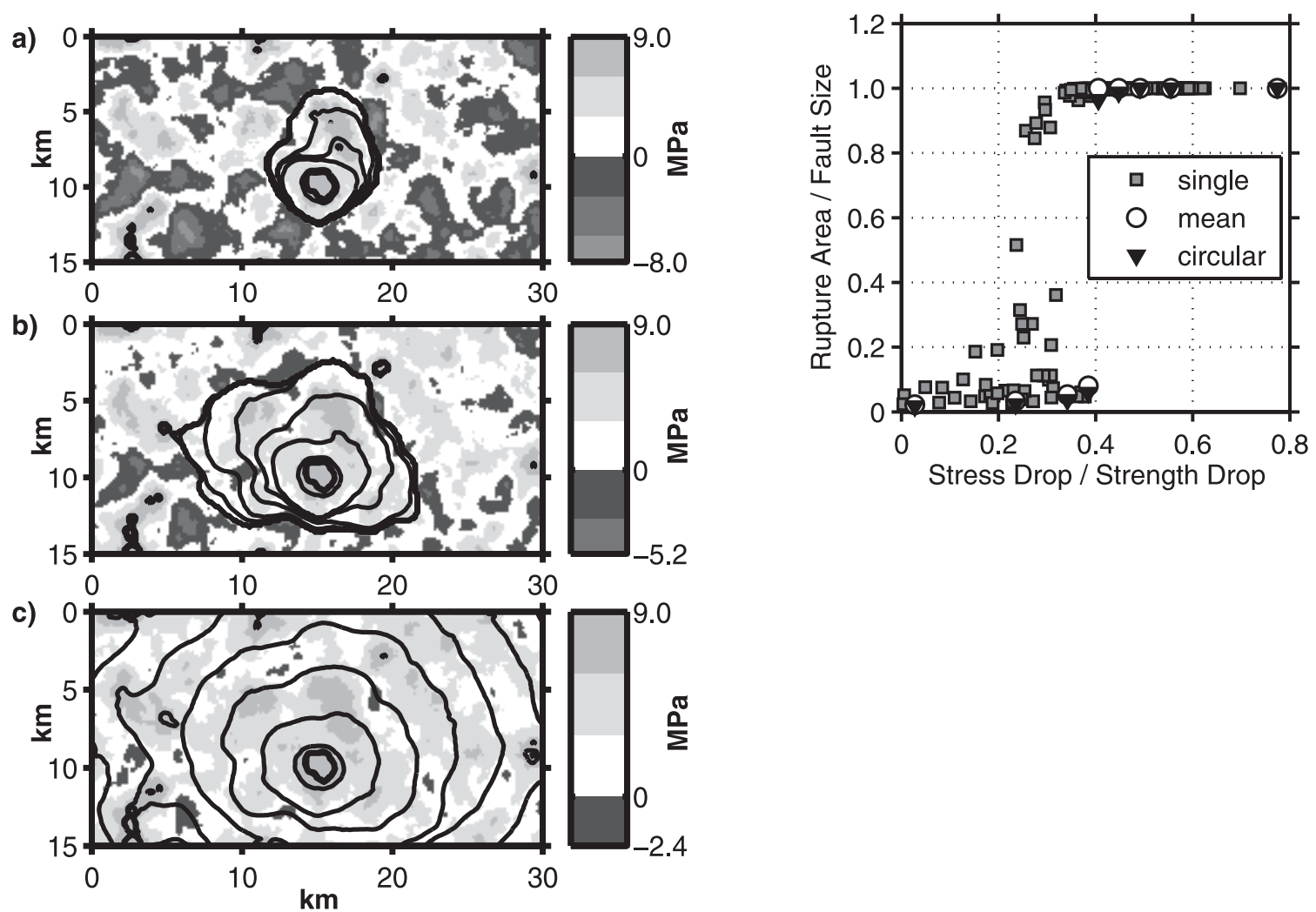

Figure 4. Properties of a collection of fully dynamic 3D rupture simulations with non uniform initial stress. (a-c) Rupture fronts for three simulations that differ only by the amplitude (std) of the available stress drop (gray scale). (right) Dependence of rupture area on fault-average stress drop (gray squares). Ruptures with small std, large stress drop, percolate through the whole fault. The transition to run-away events is well captured by dynamic simulations that start with the ensemble-averaged stress (circles) and by a mean-field analysis for circular mode I cracks (triangles).

value $s t d_{c}$ the rupture percolates through the whole fault. As shown in Figure 4-right for a large collection of dynamic calculations [Ripperger et al, 2005] this transition is well explained by the analysis proposed here, adapted to $3 \mathrm{D}$ with expressions of $K_{0}$ for circular mode I cracks averaged over the crack contour as in [Dyskin, 1999]. The present model suggests that this is a first-order, discontinuous, transition. Although our presentation has focused on the effect of $s t d$ a similar transition can be driven by an increasing correlation length at fixed $s t d$, as can be deduced from (18) noting that $m$ is a decreasing function of $a_{c}$.

The current framework can be extended in many ways but also has intrinsic limitations. At present, only bilateral, symmetric, ruptures are considered and thus the effect of directivity has not been assessed. A generalization requires an efficient determination of the crack path in a random energy landscape. The model applies to events that do not break the whole seismogenic depth, otherwise the scaling of the energy release rate $G$ should be modified. We consider only single connected cracks, although interaction and coalescence between many nucleation sites or dynamic triggering ahead of the rupture front is typically seen in our fully dynamic 3D models. Interaction between more than two cracks is hard to include. Our focus has been on cracklike ruptures, however earthquakes are often interpreted as short rise-time rupture pulses. Expressions of $G$ for pulses are available but the relation between pulse width and some characteristic healing length contained in the stress/strength heterogeneity spectrum is still poorly understood. Although important aspects of 3D dynamic rupture cannot be rendered by the present model we believe it is a useful tool to guide the analysis of more complex models.

\section{CONCLUSION}

We have introduced a semi-dynamic model of earthquake rupture, based on fracture mechanics, to study the effect of spatially correlated stress drop heterogeneities on macroscopic source parameters and general properties of seismicity. We identified a transition in magnitude-frequency statistics from Gutenberg-Richter to characteristic earthquake, driven by the amplitude of stress drop heterogeneities. 
The stress correlation length plays an important role, appearing as a characteristic short length in the scaling behavior of macroscopic source properties, and may mask or may be confused with the signature of the nucleation process.

Acknowledgments. We thank A. McGarr, R. Abercrombie and H. Kanamori for organizing the 2005 Chapman conference on Radiated Seismic Energy which provided the stimulating discussions that led to this work. J.-P. A. is grateful to T. Heaton, J. Schmittbuhl and A. Cochard for fruitful discussions. Funding for J.-P. A. was provided by SPICE, a Marie Curie Research Training Network in the 6th Framework Program of the European Commission. This is ETH contribution No. 1443 .

\section{REFERENCES}

Ampuero, J.-P., J.-P. Vilotte, and F.-J. Sánchez-Sesma (2002), Nucleation of rupture under slip dependent friction law: simple models of fault zone J. Geophys. Res., 107(B12), 10.1029/2001JB000452.

Boatwright, J. and H. Quin (1986), The seismic radiation from a 3-D dynamic model of a complex rupture process; Part I, Confined ruptures, in Earthquake source mechanics, S. Das et al (eds.), Geophysical Monograph, 37, 97-109.

Bouchbinder, E., D. Kessler and I. Procaccia (2004), Crack-microcrack interactions in dynamical fracture, Phys. Rev. E, 70(4), Art. No. 046107.

Bouchbinder, E., J. Mathiesen and I. Procaccia (2005), Branching instabilities in rapid fracture: Dynamics and geometry, Phys. Rev. E, 71(5), Art No. 056118.

Campillo, M. and I. Ionescu (1997), Initiation of antiplane shear instability under slip dependent friction. J. Geophys. Res., 102, 20363-20371.

Day, S. M. (1982), Three-dimensional simulation of spontaneous rupture: the effect of nonuniform prestress, Bull. Seism. Soc. Am., 72, 1881-1902.

Dyskin, A. V. (1999), On the role of stress fluctuations in brittle fracture, Int. J. Fract., 100, 29-53.

Guatteri, M. and P. Spudich (2000), What can strong motion data tell us about slip-weakening fault-friction laws? Bull. Seism. Soc. Am., 90, 98-116.

Heimpel, M. (1996), Earthquake size-frequency relations from an analytical stochastic rupture model, J. Geophys. Res., 101(B10), 22435-22448.

Heimpel, M. and P. Malin (1998), Aseismic slip in earthquake nucleation and self-similarity: evidence from Parkfield, California, Earth Planet Sci. Lett., 157, 249-254.
Husseini, M. I., D. B. Jovanovich, M. J. Randall and L. B. Freund (1975), The fracture energy of earthquakes, Geophys. J. Roy. Astr. Soc., 43, 367-385

Husseini, M. I. and M. J. Randall (1976), Rupture velocity and radiation efficiency, Bull. Seism. Soc. Am., 66(4), 1173-1187.

Lavallée, D., and R. J. Archuleta (2003), Stochastic modeling of slip spatial complexities from the 1979 Imperial Valley, California, earthquake. $J$. Geophys. Res., 30(5), 1245, doi:10.1029/2002GL015839.

Leise, T. L., and J. R. Walton (2001), Dynamically accelerating cracks Part 2: a finite length mode III crack in elastic material. Quart. Appl. Math., 59(4), 601-614.

Madariaga, R. (1979), On the relation between seismic moment and stress drop in the presence of stress and strength heterogeneity, J. Geophys. Res., 84(B5), 2243-2250.

Mai, P. M., and G. C. Beroza (2002), A spatial random field model to characterize complexity in earthquake slip, J. Geophys. Res., 107(B11), Art. No. 2308, doi:10.1029/2001JB000588.

Oglesby, D. D., and S. M. Day (2002), Stochastic fault stress: implications for fault dynamics and ground motion, Bull. Seism. Soc. Am., 92 , 3006-3021.

Richardson, E. and T. H. Jordan (2002), Seismicity in deep gold mines of South Africa: implications for tectonic earthquakes, Bull. Seism. Soc. Am., 92, 1766-1782.

Ripperger, J., P. M. Mai, and J.-P. Ampuero (2005), Nucleation and Propagation of Dynamic Earthquake Rupture Under Constrained Stochastic Shear Stress, Eos Trans. AGU, 86(52), Fall Meet. Suppl., Abstract S53A-1081.

Rose, L. R. F. (1976), On the initial motion of a Griffith crack, Int. J. Fracture, 12(6), 829-841.

Rundle, J., E. Preston, S. McGinnis and W. Klein (1998), Why earthquakes stop: growth and arrest in stochastic fields, Phys. Rev. Lett., 80(25), 5698-5701.

Uenishi, K., and J. R. Rice (2003), Universal nucleation length for slipweakening rupture instability under non-uniform fault loading, $J$. Geophys. Res., 108(B1), 2042, doi:10.1029/2001JB001681.

J.-P. Ampuero, Institute of Geophysics, Seismology and Geodynamics, ETH Hönggerberg, CH-8093 Zürich, Switzerland. (ampuero@erdw. ethz.ch)

J. Ripperger, Institute of Geophysics, Swiss Seismological Service, ETH Hönggerberg, CH-8093 Zürich, Switzerland. (ripperger@sed.ethz.ch)

P. M. Mai, Institute of Geophysics, Swiss Seismological Service, ETH Hönggerberg, CH-8093 Zürich, Switzerland. (martin.mai@sed. ethz.ch) 
